\title{
Methylation of DNA in Colorado potato beetle Leptinotarsa decemlineata (Say)
}

\author{
Yu.M. Nikonorov, G.V. Benkovskaya* \\ Institute of Biochemistry and Genetics, Ufa Federal Research Centre of RAS, Ufa, Russia \\ *e-mail:bengal2@yandex.ru
}

Key words: DNA methylation, Colorado potato beetle, ecdysone receptor gene

Motivation and Aim: Enzymatic methylation-demethylation of DNA is of great importance for gene activity regulation in plants and animals. For a number of investigated insect species it is typical the presence in genome of genes with both low and high levels of methylation in coding regions, that is connected to a certain extent of reducing the elements of methylation DNA systems. Our aim is to investigate the methylation status of some genes of Colorado potato beetle Leptinotarsa decemlineata and to evaluate their transcriptional activity at the separate phases of winter diapause.

Methods and Algorithms: S-adenosyl methionine (SAM) and 20-hydroxyecdysone (20E) have been applied in concentration of $1 \times 10^{-7} \mathrm{M}$ for treatment of fresh potato leaves used to feed Colorado potato beetle adults from native excerpts during 7 days. DNA and RNA extracted from tissues of thorax and gonads of adults at the stages of winter diapause initiation and diapause termination by phenol-detergent method. Relative abundance of mRNAs of genes ecdysone receptor ecr, enzyme acetylcholinesterases ache and protein transporter ferritin fer has been evaluated by qRT-PCR with reference gene $r p 18$. Level of DNA methylation in coding regions of mentioned genes determined by MSRE-PCR (methylation-sensitive restriction enzyme PCR).

Results: SAM and 20E addition to the food at the stage of diapause initiation had an adaptogenic effect under partial starvation (due to substitution of potato leaves to honey solution) and intoxication. The reducing of gene ecr mRNA ratio registered between tissues of gonads and thorax of active males and females fed by food with SAM and 20E addition.

Stimulatory action of SAM revealed toward the gene fer mRNA content in gonads of adults treated by insecticides. Content of 5-methylcytosine $(5 \mathrm{mC})$ in DNA sites of restrictase HpaII localized in investigated genes ache and fer coding regions amounted no more than $5 \%$. Proximal site against 5 '-end of ecr gene has been methylated to a greater extent than the distal site and in males it has been methylated to a greater extent than in females. SAM became apparent as an inductor of DNA demethylation process in proximal site of ecr gene in males. High correlation level $(r=0.86)$ determined between methylation of DNA level in proximal site of ecr gene and abundance of its mRNA. Conclusion: We revealed the coordinated change of methylation status of ecdysone receptor gene ecr in Colorado potato beetle adults and its transcriptional activity during the winter diapause and the sensitivity of insect to presence in the food of methyl groups. Acknowledgements: Investigations funded by RFBR No. 15-04-04801-a and 17-44020347-r_a. 